\title{
Droit des patientes et patients à l'autodétermination
}

\author{
Les principes médico-éthiques présents ont été approuvés par le Sénat de \\ I'ASSM le 24 novembre 2005. La version allemande est la version d'origine.
}

\begin{abstract}
1 Pour simplifier, nous n'emploierons que le masculin pour les deux sexes.
\end{abstract}

2 Voir glossaire à «Acte médical». La différenciation entre acte médical léger et acte médical lourd. Les exigences à poser pour que le consentement puisse être considéré comme valable dépendent principalement de la nature de l'acte médical. Ainsi, par exemple, l'information concernant une ponction veineuse est soumise à des critères moins exigeants que celle liée à une thérapie médicamenteuse susceptible d'entraîner des effets secondaires. L'information doit être aussi complète que nécessaire.

3 A l'exception de la privation de liberté à des fins d'assistance selon les art. 397a ss.

4 Voir glossaire à «Capacité de discernement».

5 Voir glossaire à «Intérêts objectifs».

6 Voir glossaire à «Représentant légal».

7 Il s'agit dans ce contexte de traitements indiqués du point de vue médical.

8 Voir glossaire à «Volonté présumée».

9 Voir glossaire à «Directives anticipées».

10 Voir glossaire à «Représentant mandaté pour les affaires médicales».

\section{Préambule}

Chaque patient ${ }^{1}$ a le droit à l'autodétermination. Donner en temps utile au patient ou à ses représentants des informations sur l'état de santé qui soient à la fois complètes, compréhensibles et nuancées en fonction de la gravité de l'acte médical $^{2}$ est indispensable pour qu'un choix éclairé puisse être fait en toute connaissance de cause (consentement éclairé). Cela suppose qu'une communication ouverte et empathique s'installe et que tous les responsables médicaux soient prêts à informer des possibilités et des limites tant de l'action curative que des soins palliatifs. Il n'en reste pas moins que le droit de tiers à disposer d'eux-mêmes peut poser des limites au droit du patient à disposer librement de sa personne: On ne doit accéder à la volonté du patient ou de ses représentants de bénéficier d'un traitement et d'une prise en charge spécifique que si ces soins correspondent aux règles communément reconnues. Par contre, le refus d'un patient capable de discernement d'être traité ou pris en charge doit être respecté ${ }^{3}$.

\section{Principes}

\section{Patient capable de discernement}

Respecter la volonté du patient capable de discernement ${ }^{4}$ est capital dans le traitement et la prise en charge. C'est pourquoi il est interdit de pratiquer tout acte médical allant à l'encontre de la volonté exprimée du patient capable de discernement. Ce principe s'applique aussi lorsque cette volonté semble être contraire aux intérêts objectifs ${ }^{5}$ du patient. La demande d'un patient souhaitant un traitement et une prise en charge spécifiques ne doit être satisfaite que si les actes demandés cadrent avec les règles de l'art médical.

Les mineurs ou les personnes sous tutelle peuvent aussi être capables de discernement lorsqu'il s'agit de consentir à un traitement.

\section{Patient incapable de discernement}

\subsection{Décision du représentant légal d'un patient n'ayant jamais été capable de discernement}

Si le patient n'est pas capable de discernement et qu'il ne l'a jamais été auparavant, le représentant légal ${ }^{6}$ donne son consentement au traitement et à la prise en charge. En ce qui concerne les enfants et les adolescents incapables de discernement, cette tâche incombe en règle générale aux parents. Le représentant doit respecter les intérêts objectifs du patient lorsqu'il prend sa décision. Par conséquent, le refus d'un traitement et d'une prise en charge indispensables d'un point de vue médical n'est pas autorisé7.

Les décisions touchant à la vie et à la mort représentent, en particulier pour les parents, une tâche lourde et parfois à peine supportable. Ils ne devraient donc être associés au processus décisionnel que dans la mesure où ils le souhaitent. Les décisions relatives au traitement et à la prise en charge doivent être prises dans l'intérêt objectif de l'enfant et en accord avec ses parents ou d'autres représentants légaux.

\subsection{Recherche de la volonté présumée du patient ayant été antérieurement capable de discernement}

Si le patient a été capable de discernement à une période antérieure et qu'il n'est plus en mesure d'exprimer ses souhaits, on doit s'enquérir de sa volonté présumée ${ }^{8}$. A ce propos, il convient avant tout de rechercher s'il a personnellement manifesté sa volonté par écrit sur ce sujet (directives anticipées ${ }^{9}$ ). Il importe également de prendre en compte les déclarations d'une personne désignée par le patient et mandatée pour les questions médicales (représentant thérapeutique $^{10}$ ). Il appartient dans le même temps au médecin et au personnel soignant de déterminer si le patient a clairement exprimé sa volonté 
d'une autre manière, notamment vis-à-vis de ses proches. En principe, la volonté présumée du patient, que l'on déduit de directives anticipées, de déclarations d'un représentant thérapeutique ou à partir d'autres indices, a la priorité sur toute décision contraire du représentant légal ${ }^{11}$.

\section{Directives anticipées}

Toute personne peut déterminer à l'avance quel traitement et quelle prise en charge elle souhaite subir au cas où elle deviendrait incapable de discernement. Les directives anticipées doivent être respectées dans la mesure où elles portent sur un traitement médicalement indiqué ou sur le refus d'un traitement et qu'elles correspondent à la situation concrète dans laquelle se trouve le patient, pour autant qu'il n'existe pas d'indices permettant de penser que la volonté du patient a changé entre-temps. En cas de maladie prévisible, le médecin a le devoir de rendre le patient attentif à la possibilité de rédiger des directives anticipées.

11 Sous réserve de dispositions cantonales divergentes. Les cantons du Jura, du Tessin et de Neuchâtel prévoient par exemple qu'une personne proche ou un membre de la famille peuvent donner leur consentement pour une personne majeure incapable de discernement. Les cantons d'Argovie, d'Appenzell Rh.-Ext., de Berne, de Lucerne et de Zurich accordent, pour leur part, au médecin le droit de prendre des décisions après s'être entretenu avec les proches. Dans les cantons de Bâle-Ville et de Genève, on doit faire appel à l'autorité tutélaire.

12 Actuellement, à Bâle-Ville et à Genève, on est en principe obligé de faire appel à l'autorité tutélaire.

13 Voir glossaire à «Urgence de la situation».

14 Dans ce contexte, les «commissions d'éthique clinique» s'entendent comme un terme général comprenant les différentes formes de soutien dans les questions médico-éthiques, comme par ex. les consultations d'éthique, les consultations par une commission d'éthique ou les commissions ad hoc etc.

15 Convention pour la protection des Droits de l'homme et de la dignité de l'être humain à l'égard des applications de la biologie et de la médecine: Convention sur les Droits de l'Homme et la biomédecine. Oviedo 1997.

16 Cf. en particulier Beauchamp T L, Childress J F. Principles of Biomedical Ethics. 2001.

\section{Représentant thérapeutique}

Toute personne peut nommer à l'avance et par écrit un représentant mandaté pour les affaires médicales (représentant thérapeutique). Celui-ci est habilité à consentir à un traitement ou à le refuser à la place du patient lorsque celui-ci se trouve dans un état d'incapacité de discernement. La décision doit être prise en tenant compte des directives anticipées existantes et en accord avec le représentant thérapeutique désigné (cf. chiffre 3. Situations conflictuelles).

\section{Autres indices de la volonté présumée}

Il n'est pas rare qu'il n'existe ni directives anticipées valables, ni représentant thérapeutique. Dans ce cas, on doit alors rechercher des informations sur les convictions et les comportements antérieurs du patient. S'entretenir avec les proches et toute autre personne concernée, tel le médecin de famille, permet en général d'obtenir de précieux renseignements.

\section{Prise de décision dans un esprit de consensus} A chaque fois que cela est possible, les décisions doivent être prises dans un esprit de consensus entre les responsables médicaux, les représentants et les proches. Cela signifie qu'il faut tout tenter pour que les différentes parties réussissent à s'entendre tant sur la volonté présumée que sur l'intérêt objectif du patient. Cela constitue le meilleur moyen de prendre en compte d'une part les meilleures connaissances médicales du personnel soignant et d'autre part le rapport plus personnel que peuvent avoir notamment les proches avec le patient. Le contrôle des décisions médicales s'en trouve renforcé tout comme la confiance du public dans la médecine.

C'est seulement dans le cas où les différentes parties ne peuvent arriver à un accord que l'on fait appel à l'autorité tutélaire, sous réserve de dispositions cantonales divergentes ${ }^{12}$.

\subsection{Recherche de l'intérêt objectif du patient}

Il s'avère parfois impossible de trouver des indices concernant la volonté présumée du patient, surtout lorsque l'urgence de la situation ${ }^{13}$ ne laisse pas le temps de consulter des tierces personnes. Dans de telles situations, la décision des responsables médicaux chargés du traitement ou de la prise en charge doit être prise en fonction des intérêts objectifs du patient.

\section{Situations conflictuelles}

Si le représentant (légal ou thérapeutique) refuse un acte médical étant nécessaire d'un point de vue médical pour sauver la vie du patient incapable de discernement ou pour le prévenir de complications graves, toutes les possibilités de médiation, comme par ex. le recours à une commission d'éthique clinique ${ }^{14}$, doivent être utilisées. En l'absence d'accord, on fera appel à l'autorité tutélaire. En situation d'urgence, lorsqu'il n'y a pas assez de temps pour respecter ces différentes étapes, une mesure de ce type peut être prise même contre la volonté du représentant.

\section{Commentaire}

Le groupe de travail a pris en premier lieu comme base de réflexion les dispositions du droit fédéral et la liberté de décision qu'il permet. Par ailleurs, il a tenu compte de la convention bioéthique européenne ${ }^{15}$ ainsi que des principes généraux de l'éthique médicale ${ }^{16}$. A l'échelon cantonal, il existe actuellement différentes réglementations qui semblent en partie incompatibles avec le droit fédéral (par ex. Bâle-Ville qui donne la priorité à l'option basée sur le droit de l'autorité tutélaire ou certains cantons romands qui accordent une valeur particulièrement grande aux directives anticipées). Avoir des réglementations qui diffèrent d'un canton à l'autre n'est ni satisfaisant, ni rassurant pour les patients et les équipes médicales. Et ceci d'autant plus que pour des actes médicaux importants, les patients sont hospitalisés dans un autre canton que le leur (ce qui va probablement être de plus en plus le cas) et qu'ils sont donc soumis à des réglementations «hors canton». Pour cette raison, le groupe de travail considère qu'il est 
urgent d'adopter une réglementation fédérale uniforme.

En ce qui concerne l'importance des directives anticipées en particulier, mais aussi de la prise de décisions consensuelles en général, le groupe de travail est arrivé à des résultats quelquefois controversés dans les ouvrages juridiques et éthiques. Il considère toutefois que sa vision des choses est fondée.

\section{Ad 1. (Patient capable de discernement)}

Le traitement et la prise en charge de patients capables de discernement ne sont légitimes que s'il existe un consentement valable; la condition sine qua non de la validité du consentement est l'information préalable sur l'acte médical et sa portée (risques, conséquences positives, alternatives, etc.).

\section{Ad. 2. (Patient incapable de discernement)}

Pour les actes médicaux sur des personnes incapables de donner elles-mêmes leur consentement, on doit, par la force des choses, se baser sur des consentements de substitution. Les déclarations pouvant se substituer au consentement du patient sont: une décision de l'autorité tutélaire, un consentement écrit rédigé à une date antérieure («directives anticipées»), un consentement donné par des représentants légaux ou par un représentant mandaté par le patient, ou un consentement présumé.

\section{Ad. 2.1.}

S'il s'agit de personnes qui n'ont jamais été capables de discernement auparavant (incapacité de discernement originaire), tous les consentements de substitution rattachés à une capacité de discernement antérieure sont exclus. Tel est le cas du représentant désigné par le patient ou des directives anticipées. Dans ces circonstances, seuls restent possibles le recours à la représentation légale exercée par les parents ou les tuteurs, de même que les (autres) instruments du droit tutélaire. Le représentant légal doit dans tous les cas avoir comme préoccupation principale le bien objectif, c'est-à-dire défini d'un point de vue médical, de la personne qu'il représente. En ce qui concerne les questions médicales, il ne peut consentir qu'à des actes médicaux indiqués et ne peut pas refuser des actes médicaux indiqués ou exiger des actes médicaux non indiqués.

\section{Ad. 2.2 .}

Dans tous les autres cas, c'est-à-dire lorsqu'une phase de capacité de discernement a précédé la phase d'incapacité (incapacité de discernement acquise), on peut en principe se baser sur tous les consentements de substitution cités plus haut. La question qui se pose alors en priorité est celle de leur hiérarchisation dans les situations conflictuelles.

Pour ce qui est de la hiérarchisation des consentements de substitution, le principe de subsidiarité valable pour le droit tutélaire devrait avoir pour conséquence qu'une décision de l'autorité tutélaire (c'est-à-dire en particulier la désignation d'un curateur ou, si le temps presse, une décision de cette même autorité dans une situation concrète) n'entre en ligne de compte que lorsque d'autres consentements de substitution ne remplissent pas leur tâche ou qu'il existe un conflit d'intérêts ou d'opinions.

Cela dit, pour une partie de la doctrine juridique, les dispositions du droit tutélaire prennent le pas sur un représentant désigné par le patient lorsque se développe une incapacité durable de discernement. Dans certains cas, on accepte également, selon les circonstances, une combinaison de ces dispositions avec une représentation désignée par le patient (on peut par ex. proposer de laisser au représentant désigné par le patient le soin de s'occuper de la question médicale sous la surveillance de l'autorité). Le principe de subsidiarité du droit tutélaire se trouve encore plus limité lorsqu'est posé comme postulat général le recours à l'autorité tutélaire au cas où le patient incapable de discernement n'a pas de représentant légal. (A tire d'exemple, ce cas est prévu dans l'ordonnance relative à la loi sur l'hôpital de Bâle-Ville ${ }^{17}$ ). Elle stipule ainsi la priorité aux instruments du droit tutélaire, même par rapport à une représentation définie par le patient lui-même ou à des directives anticipées.

La pratique montre que vouloir faire intervenir en premier lieu l'autorité tutélaire pour des patients incapables de discernement n'est pas réalisable, car des décisions importantes sur le plan médical doivent souvent être prises dans des situations où le temps presse (sans qu'il s'agisse pour autant de cas d'urgence). Il arrive aussi souvent que les ressources en personnel des autorités tutélaires ne leur permettent pas d'assumer pleinement la fonction de représentant. Dans ces conditions, le personnel médical traitant reçoit l'instruction informelle de prodiguer des soins en fonction de la volonté présumée du patient. D’un point de vue médical, l'idéal est d'établir une hiérarchie des consentements de substitution qui soit la plus homogène et la plus pratique possible. On devrait pouvoir faire appel à l'autorité tutélaire dans les situations conflictuelles. 
La jurisprudence du Tribunal fédéral laisse en tout cas aux cantons la liberté d'habiliter les représentants désignés par le patient ou les personnes proches à prendre des décisions et de s'écarter ainsi de la voie du droit tutélaire. L'importance du droit tutélaire est également atténuée en pratique dans la mesure où une exigence formelle de consentement de la part d'un représentant légal se limite souvent, par le biais du droit de la santé cantonal, à des décisions fondamentales liées à un traitement, à des opérations comportant des risques, à des thérapies onéreuses et à la planification de méthodes de traitement pour des maladies graves qui dureront probablement très longtemps. A cela s'ajoute le fait que l'on prend bien comme point de départ la volonté présumée.

L'élément qui est particulièrement sujet à controverse est l'importance des directives anticipées. Pour les uns, elles expriment la volonté du patient que l'on doit absolument respecter. Pour les autres, elles constituent (seulement) un indice important lorsque l'on recherche la volonté présumée du patient.

La question n'a jusqu'à présent pas fait l'objet d'une réglementation spéciale au niveau fédéral. Reste que dans le cadre de la révision de la protection de l'adulte, on relève des tentatives pour inscrire les directives anticipées dans la loi (voir art. 373 de l'avant-projet de juin 2003: «Des directives anticipées suffisamment précises valent consentement ou refus de traitement lors de la survenue de la situation envisagée par leur auteur. A défaut, elles donnent des indications à son représentant ou, en cas d'urgence, à son médecin traitant.»).

Actuellement, il existe des prescriptions différentes au niveau cantonal. Certains cantons accordent une importance propre aux directives anticipées, d'autres, en revanche, considèrent qu'elles sont l'expression de la volonté présumée du patient et qu'elles doivent être prises en compte. La Convention du Conseil de l'Europe sur les droits de l'Homme et la biomédecine, qui devrait être prochainement ratifiée par la Suisse, ne part pas du principe que les directives anticipées revêtent un caractère obligatoire exclusif, abstraction faite d'autres indices. Elle stipule dans l'art. 9: «Les souhaits précédemment exprimés au sujet d'un acte médical par un patient qui, au moment de l'acte médical, n'est pas en état d'exprimer sa volonté seront pris en compte.» Mis à part le fait que les souhaits exprimés antérieurement peuvent rarement porter sur l'acte médical qui entre en ligne de compte ultérieurement, même dans les grandes lignes («au sujet d'un acte médical»), une prise en compte de la volonté exprimée antérieurement par le patient peut être garantie même lorsque l'on tient compte d'éventuels autres indices existants.

Les avis concordent pour les situations d'urgence. On doit, en principe, prendre en premier lieu les mesures médicalement indiquées. Les directives anticipées n'entrent en jeu qu'en second lieu.

La diversité des positions au sujet de la valeur des directives anticipées se reflète dans les arguments exposés ci-dessous:

\section{Les directives anticipées comme expression} des souhaits du patient à respecter absolument

- Rédiger des directives anticipées revient à faire valoir son droit à l'autodétermination. On doit donc considérer les directives anticipées comme l'expression de la volonté du patient à respecter absolument (à moins que des raisons majeures ne s'y opposent: capacité de discernement défaillante, changement d'opinion manifeste). Le risque que la décision prise ait été différente dans la situation concrète que lors de la rédaction des directives est alors supporté par le patient.

- Le «consentement antérieur» exprimé dans le cadre des directives anticipées vient après le consentement présent. Il doit par conséquent figurer de toute façon en premier dans la liste des consentements de substitution pour les personnes devenues incapables de discernement qui n'ont pas de représentant légal.

- A l'argument selon lequel des souhaits exprimés antérieurement ne devraient pas être assortis d'exigences moins importantes que les souhaits exprimés le moment venu, on oppose le fait que même le patient capable de discernement peut refuser toute information et laisser au personnel médical le soin de prendre la décision le concernant.

\section{Les directives anticipées comme indice essentiel} lors de la recherche de la volonté présumée

- Les directives anticipées constituent des dispositions préalables en vue d'une situation existentielle difficilement prévisible. On ne peut donc pas les comparer, ni d'un point de vue juridique ni d'un point de vue éthique à une déclaration du patient capable de discernement faite le moment venu.

- Les consentements exprimés antérieurement ne doivent pas être assortis d'exigences moins importantes que les consentements donnés dans la situation même. On ne peut parler en principe de consentement valable que lorsque le patient a reçu une information suffisante. 
Or les directives anticipées ne répondent souvent pas à ce critère (dans la plupart des cas, le patient rédige des directives anticipées sans avoir été informé; même lorsqu'il l'a été, il n'est pas rare que, le temps passant, ces directives ne correspondent plus forcément à la situation présente, et même si elles y correspondent encore, il était impossible, au moment où elles ont été rédigées, d'anticiper le tableau clinique exact avec les bénéfices et les risques concrets de tel ou tel acte médical). En d'autres termes, lorsque le patient rédige des directives anticipées, il ne peut pas se projeter dans l'avenir par rapport à son état de santé, aux différentes décisions possibles et aux alternatives qui lui seront proposées.

En fin de compte, les deux points de vue ne sont pas si différents qu'on pourrait le croire au premier abord, car les directives anticipées nécessitent généralement une interprétation. On doit en outre vérifier s'il existe des indices démontrant que les directives anticipées ont été annulées ou que le patient a changé d'avis.

Du point de vue du groupe de travail, les directives anticipées constituent un indice essentiel lors de la recherche de la volonté du patient. La «relativisation» de son importance ne revient pas, selon le groupe de travail, à limiter l'autonomie du patient, mais permet, bien au contraire, de la garantir, également et précisément au moment de la prise en charge et du traitement. Lorsque l'on doit en particulier prendre des décisions existentielles et irréversibles concernant un patient en fin de vie, comme arrêter les mesures destinées à le maintenir en vie, les directives anticipées ne peuvent pas décharger complètement l'équipe soignante de la responsabilité finale. On sait par expérience que les personnes ont un regard plus positif sur une vie marquée par la maladie et la nécessité de suivre des soins quand elles sont elles-mêmes dans cette situation-là, qu'elles ne l'avaient auparavant lorsqu'elles étaient en bonne santé. Partant de cette constatation, il est tout à fait possible que l'on soit obligé dans certaines situations de vérifier si le patient n'aurait peutêtre pas pris une autre décision après avoir été informé de son état. Le groupe de travail estime par conséquent que l'on doit tout autant respecter, après la perte de la capacité de discernement, la volonté naturelle perceptible dans la situation de traitement et de prise en charge. Cette volonté peut aussi se manifester (de manière non verbale) dans les gestes et les com- portements et être éventuellement en opposition avec des vœux exprimés dans les directives anticipées.

Mais ces limitations et relativisations ne permettent en aucun cas de décider, selon sa propre appréciation, de prendre en compte ou non les directives anticipées. Plus des directives anticipées sont claires, plus elles s'appliquent de façon concrète à l'état médical en question, plus leur rôle dans le processus de décision est important (p.ex. dans le cas d'un malade du Sida qui s'est informé très précisément sur les différents stades de la maladie et qui prend une décision en conséquence à l'avance). Par contre, si les directives anticipées sont formulées en termes plus généraux, elles expriment davantage l'échelle des valeurs du patient que la manifestation de sa volonté applicable dans un cas concret.

La question qui se pose aussi souvent est de savoir à qui incombe la tâche de se renseigner sur l'existence de directives anticipées. Le groupe de travail juge souhaitable que l'équipe soignante demande systématiquement au patient, au moment de son admission à l'hôpital, s'il a rédigé des directives anticipées et que le médecin, notamment en cas de maladie prévisible, le rende attentif à la possibilité de rédiger des directives anticipées. Il serait tout aussi judicieux de conserver les directives anticipées des patients dans un endroit centralisé, tel le service d'urgence médical du canton.

La décision prise par un représentant désigné par le patient (lequel est soit désigné directement en tant que représentant pour les questions médicales, soit appelé à titre de tuteur ou de curateur obligatoire désigné préalablement - art. 381 CC - dans des «directives concernant la prise en charge») se rapproche dans sa portée des directives anticipées. Elle constitue un indice (fort) lors de la recherche de la volonté présumée du patient le moment venu, mais elle ne peut pas non plus revêtir un caractère obligatoire exclusif pour les raisons mentionnées à propos des directives anticipées. Il n'empêche qu'elle se distingue de la représentation légale en ce qu'elle résulte du droit à l'autodétermination du patient.

Voilà pourquoi, dans les cas d'incapacité de discernement acquise du patient et d'un désaccord entre le représentant désigné par le patient (alors qu'il était encore capable de discernement) et un représentant légal (désigné ultérieurement), la personne autorisée par le patient devrait avoir la préférence (idée fondamentale du principe de subsidiarité). On fera des exceptions 
à ce niveau si la capacité de discernement est durablement perdue. Une partie de la doctrine considère en effet que la procuration donnée au représentant par le patient prend fin lorsque celui-ci perd durablement la capacité de discernement (voir plus haut dans le texte pour de plus amples détails).

Lorsque son consentement est requis, le représentant désigné par le patient doit, tout comme le représentant légal, se préoccuper avant tout $\mathrm{du}$ bien du patient. Il ne peut donc consentir qu'à des actes médicaux indiqués. Il a toutefois le droit, si le patient le lui a demandé, de refuser des actes médicaux indiqués et indispensables d'un point de vue médical (dans cette situation conflictuelle, le personnel médical peut s'adresser à l'autorité tutélaire).

Pour toutes ces raisons, le consentement présumé du patient prend une importance capitale, en particulier lorsque l'on considère les directives anticipées comme un indice (essentiel) dans le cadre d'un consentement présumé, que le représentant désigné doit en tous les cas agir pour le bien du patient au moment de consentir à des actes médicaux et que les instruments du droit tutélaire n'ont qu'une fonction subsidiaire.

En ce qui concerne le consentement présumé, on doit dans ces conditions décider qui est habilité en premier lieu à recueillir la volonté présumée. Etant donné que la constatation de cette volonté suppose aussi à chaque fois que l'on connaisse bien les souhaits personnels du patient et les composants objectifs (c'est-à-dire: à définir par le corps médical) du «bien», le groupe de travail propose de privilégier à ce niveau une décision prise par consensus entre les proches et le personnel médical traitant.

Ceci ne limite pas du tout le droit à l'autodétermination, mais le rend en fait - à peu près - applicable pour les patients incapables de discernement. Le contrôle mutuel de deux groupes, chargés de définir la volonté présumée du patient, est le meilleur moyen de garantir le respect de cette volonté. Il peut, mieux que tout autre dispositif de prise de décision, empêcher que des préférences personnelles ne prennent le pas sur le respect de la volonté présumée du patient. $\mathrm{Si}$, au contraire, on donne uniquement la priorité aux directives anticipées ou au représentant désigné, on considère alors comme valable, en contradiction avec le système, un consentement qui a été donné sans information préalable au patient sur l'acte médical concret à mener ou à ne pas mener.

L'idée de la priorité accordée à une décision consensuelle se retrouve aussi dans les principes défendus par la doctrine juridique: le personnel soignant doit se prononcer sur les indications médicales, les proches sur les indications individuelles et seuls les deux types d'indication réunis rendent l'acte médical légitime. Même l'idée d'une séparation entre la décision et l'acte médical, pour des raisons de contrôle, parle pour une forme consensuelle de ce type.

Cela dit, si les différents acteurs n'arrivent pas à s'entendre, la décision doit être renvoyée à l'autorité tutélaire. Mais c'est bien là que réside toute sa fonction.

La conséquence logique de ce genre de réglementation par consensus serait que le secret médical vis-à-vis des proches participant aux décisions n'existe pas - juridiquement parlant, on devrait sans doute considérer la défense d'intérêts légitimes comme fait justificatif. Pour ce qui est de la réglementation relative à la responsabilité, le médecin continuera à assumer seul la responsabilité en cas d'erreur de comportement du corps médical, pour autant que ses indications aient été suivies. Dès que ceci n'est pas l'objet du consensus et que le médecin s'adresse pour cette raison à l'autorité tutélaire, celle-ci est de toute façon obligée de prendre la décision et elle décharge par là même le médecin et les proches de toute responsabilité.

\section{Glossaire}

\section{Acte médical}

\section{Acte médical léger}

Acte qui ne présente aucun risque ou des risques limités pour le patient (par ex. prélèvement de sang à des fins diagnostiques ou mesures de soins ou thérapeutiques telles que l'hygiène corporelle).

\section{Acte médical lourd}

Actes médicaux invasifs sur le corps (opération, chimiothérapie, traitement par rayons, etc.) et mesures permettant de maintenir le patient en vie, qui présentent un risque élevé en termes d'effets secondaires indésirables, de séquelles ou de menace pour la vie ou qui sont lourds pour le patient.

En ce qui concerne les exigences liées à l'information, sont également qualifiés de lourds les actes médicaux qui ne sont pas clairement indiqués d'un point de vue médical, parce qu'il existe plusieurs traitements possibles ou que la décision en faveur ou contre un acte médical dépend de l'attitude fondamentale d'un patient (par ex. dans le domaine de la prise en charge en fin de vie). 
La délimitation entre acte médical lourd et acte médical léger n'est pas toujours facile dans les cas concrets car les transitions de l'un à l'autre sont floues.

Bien du patient/intérêts objectifs du patient En raison des valeurs générales et objectives découlant de l'ordre juridique (par ex. droit à la vie, etc.), tout traitement médical doit être motivé par le souci objectif de guérir et de soulager le patient (indication médicale pour un traitement).

\section{Capacité de discernement}

Un consentement n'est considéré comme valable que si le patient est capable de discernement. Cette capacité est appréciée selon la situation concrète et pour l'acte concret. Elle suppose, d'une part, que le patient qui donne son consentement est en mesure d'apprécier raisonnablement la signification, l'opportunité et la portée d'une action déterminée (élément intellectuel) et, d'autre part, qu'il ait la faculté d'agir librement sur la base de cette appréciation (élément caractériel). Il n'y a pas de solutions schématiques.

En principe, la capacité de discernement est présumée. Toutefois, en cas de doute, les critères énumérés ci-dessous permettent d'apprécier la capacité de discernement:

- la capacité de comprendre les informations concernant la décision à prendre;

- la capacité d'évaluer correctement la situation et les conséquences que pourraient avoir les différentes options envisageables;

- la capacité d'évaluer des informations de manière rationnelle dans le contexte d'un système de valeurs cohérent;

- la capacité d'exprimer librement ses propres choix.

On distingue deux groupes de personnes incapables de discernement en fonction de la nature et de l'origine de leur incapacité:

Incapacité de discernement véritable ou originaire Il s'agit de patients qui ne sont pas capables, depuis leur naissance, de faire preuve d'une quelconque volonté leur permettant d'agir en conséquence. C'est le cas des jeunes enfants et des personnes handicapées mentales.

Incapacité de discernement acquise ou ultérieure On parle d'incapacité ultérieure lorsque les patients ont perdu la capacité de discernement dont ils étaient dotés auparavant. On fait en outre la distinction entre incapacité de discernement passagère (par ex. perte de connaissance consécutive à un accident) et incapacité de discernement durable (due, par ex., à une maladie neurodégénérative).

$\rightarrow$ Voir aussi pour plus d'informations:

Art. 11 Constitution fédérale (protection des enfants et des jeunes): Les enfants et les jeunes «exercent leurs droits dans la mesure où ils sont capables de discernement»

Art. 16 Code Civil Suisse: «Toute personne qui n'est pas dépourvue de la faculté d'agir raisonnablement à cause de son jeune âge, ou qui n'en est pas privée par suite de maladie mentale, de faiblesse d'esprit, d'ivresse ou d'autres causes semblables, est capable de discernement dans le sens de la présente loi.»

\section{Consentement libre et éclairé}

Décision éclairée du patient (ou de son représentant) consentant à un traitement et à une prise en charge (ou les refusant). Pour que le consentement soit libre et éclairé, il faut non seulement que le patient soit capable de discernement et que sa volonté soit librement exprimée, mais aussi qu'une information appropriée relative au diagnostic, au pronostic et aux différents traitements possibles, au traitement recommandé et aux chances/risques qu'il comporte lui soit communiquée. Le consentement éclairé suppose également que l'on s'assure que l'information ait été suffisamment comprise par le patient. En règle générale, le personnel médical doit fournir des informations plus détaillées en cas d'acte médical lourd. Le patient doit disposer d'un laps de temps suffisant pour se décider et il doit être informé de la possibilité de révoquer sa décision.

\section{Directives anticipées}

Déclaration écrite par laquelle une personne capable de discernement au moment de sa rédaction indique, au cas où elle perdrait postérieurement cette capacité, quel traitement et quelle prise en charge elle accepte ou refuse dans une situation médicale déterminée et mentionne si elle souhaite mandater une personne pour prendre à sa place les décisions ou pour consentir (de façon complémentaire) ( $\rightarrow$ procuration pour des décisions médicales).

\section{Proches}

Sont considérés comme proches le conjoint ou le partenaire, les parents ainsi que les personnes qui ont les liens les plus étroits avec le patient. Il se peut que des prescriptions cantonales définissent la notion de «proches» d'une autre façon. 


\begin{abstract}
Indications concernant
I'élaboration de ces principes

Mandat

Le 12 décembre 2003, la Commission Centrale d'Ethique de l'ASSM

a chargé un groupe de travail

d'élaborer des principes médico-

éthiques concernant le droit des

patientes et patients à l'autodéter-

mination.
\end{abstract}

Groupe de travail responsable

Prof. Kurt Seelmann,

droit (Président), Bâle

Dr Martin Conzelmann,

gériatrie, Bâle

Dr Barbara Federspiel,

médecine interne, Zurich

Prof. Annemarie Kesselring, science des soins infirmiers, Bâle

Prof. Dieter Ladewig,

psychiatrie, Bâle

Dr iur. Jürg Müller, droit, Bâle

Prof. Rudolf Ritz,

médecine intensive, Bâle

Lic. iur. Michelle Salathé,

ASSM, Bâle

Experts consultés

Avv Hp. Kuhn, droit, Berne

Prof. Audrey Leuba,

droit, Neuchâtel

Dr Marc Thommen, droit, Bâle

Lic. iur. Barbara Pfister, droit, Bâle

Approbation

Les principes médico-éthiques

présents ont été approuvés par

le Sénat de l'ASSM le 24 novembre

2005

Adresse de commande

ASSM

Petersplatz 13

CH-4051 Bâle

Tél.: 0612699030

Fax: 0612699039

mail@samw.ch

Toutes les directives médico-

éthiques de l'ASSM sont disponibles

sur www.assm.ch.
De par leur devoir général d'assistance fixé par la loi, les conjoints (art. 159 CC), les parents et les enfants (art. 272 CC) sont tenus d'une certaine manière de prendre part aux décisions médicales. Ils ont le droit d'être entendus et influent par là même sur la prise de décision. Mais ils n'ont toutefois pas de pouvoir de décision direct par rapport au médecin, à moins que le droit cantonal ne le prévoie expressément (comme c'est le cas, par exemple, dans les cantons du Jura, du Tessin et de Neuchâtel).

Si le patient a plusieurs proches et qu'ils sont d'un avis contraire, il peut être utile de procéder à la hiérarchisation suivante:

1. le conjoint ou le partenaire vivant avec le patient ou provisoirement hors du ménage;

2. la personne, parmi les descendants, parents ou frères et sœurs de la personne incapable de discernement, qui a la relation la plus étroite avec le patient, notamment parce qu'elle vit sous le même toit que la personne incapable de discernement ou qu'elle l'assiste personnellement de manière régulière (projet CC art. 434).

Les proches ne sont autorisés à obtenir des informations sur l'état de santé d'un patient que si celui-ci a donné son accord.

Si le patient n'est plus en mesure de s'exprimer sur ce sujet, on pourra supposer qu'il accepte que l'on donne des renseignements aux personnes les plus proches, sauf si les circonstances laissent penser que le patient souhaite le respect du secret médical.

\section{Représentant légal}

Personne qui, en raison du pouvoir qui lui est conféré par la loi, représente le patient. Les représentants légaux sont les parents pour l'enfant (art. 304 CC), le tuteur pour le patient mineur qui n'est pas sous l'autorité parentale (art. 368 CC) et pour le patient incapable de discernement (art. 369 CC).

$\rightarrow$ Voir aussi: art. 304 CC

$~^{1}$ Les père et mère sont, dans les limites de leur autorité parentale les représentants légaux de leurs enfants à l'égard des tiers.

${ }^{2}$ Lorsque les père et mère sont tous deux détenteurs de l'autorité parentale, les tiers de bonne foi peuvent présumer que chaque parent agit avec le consentement de l'autre. [...]»
Représentant mandaté pour les questions médicales (représentant thérapeutique)

\section{$\rightarrow$ procuration}

Personne désignée au préalable par le patient capable de discernement. Elle est chargée, pour une situation médicale déterminée, de consentir, à la place du patient devenu incapable de s'exprimer personnellement, à des mesures médicales, de soins et/ou thérapeutiques. Le patient peut donner des instructions concernant l'exercice du droit de consentement.

Le médecin traitant est libéré de son obligation de garder le secret vis-à-vis du représentant thérapeutique désigné par le patient; pour plus de sûreté, ceci devrait être expressément notifié dans la procuration.

\section{Urgence de la situation}

Acte médical (traitement, soins ou prise en charge) à pratiquer de toute urgence pour sauver la vie du patient ou prévenir toute complication grave, auquel ni le patient lui-même, ni son représentant ne peuvent consentir à temps, ou lorsqu'il existe des doutes fondés sur la validité du refus d'un traitement en raison de l'incapacité à discerner du patient ou à cause de la collision des intérêts avec le représentant légal. L'acte médical doit être apte à atteindre le but escompté sur l'état de santé du patient et doit être proportionné.

\section{Volonté présumée}

Elle consiste à se demander quelle décision prendrait un patient qui n'est plus en mesure de s'exprimer lui-même s'il pouvait le faire (volonté individuelle présumée du patient concerné). Cette volonté présumée découle de l'évaluation de toutes les informations que l'on peut recueillir comme les directives anticipées, les déclarations faites antérieurement et d'autres indications biographiques.

Ce n'est que lorsque l'on ne trouve aucune indication sur la volonté présumée individuelle que l'on va se baser sur une volonté supposée, établie à partir de critères objectifs $(\rightarrow$ Bien du patient/intérêts objectifs du patient). 